\title{
High sensitivity absorption measurement of small metal clusters embedded in an argon matrix
}

\author{
F. Conus, J. T. Lau, ${ }^{\text {a) }}$ V. Rodrigues, ${ }^{\text {b) }}$ and C. Félix \\ Institut de Physique des Nanostructures, École Polytechnique Fédérale de Lausanne (EPFL), \\ CH-1015 Lausanne, Switzerland
}

(Received 18 May 2006; accepted 26 September 2006; published online 14 November 2006)

\begin{abstract}
We describe a new UV-Vis absorption setup designed to measure with improved sensitivity the absorption spectra of size-selected small metal clusters embedded in rare gas matrices. We aim at studying the electronic structure of clusters of different materials and their interaction with the matrix. The design, construction, and performance of the setup are discussed and demonstrated on the absorption spectrum of $\mathrm{Ag}_{1}$ in argon. The absorption spectra for the two least abundant silver cluster ions produced by our cluster source in the size range of 1-39 atoms/cluster, namely, $\mathrm{Ag}_{6}$ and $\mathrm{Ag}_{10}$, are reported. A sensitivity increase of about 20 is demonstrated. (C) 2006 American Institute of Physics. [DOI: 10.1063/1.2369640]
\end{abstract}

\section{INTRODUCTION}

A very appealing feature of nanoscopic systems is the possibility to tune their properties as a function of their size. There is therefore a strong interest in understanding the size evolution of the electronic structure of these objects. Atomic nanoclusters are of particular interest since their atomic arrangement and electronic structure change when adding or removing a single atom. To investigate them, a variety of experimental techniques using mass separation has been used, ${ }^{1-10}$ while from the theory side, these systems are sufficiently simple to allow for state-of-the-art quantum mechanical calculations. ${ }^{11}$ Such size-selected studies constitute therefore benchmark systems for the understanding of nano-objects.

In the case of metals, where the electronic structure of bulk material close to the Fermi energy does not show energy gaps, small metal clusters always show discrete electronic levels. ${ }^{12}$ For this reason, small metal clusters show interesting properties: luminescence, ${ }^{7,9,13,14}$ strong catalytic activity, ${ }^{6,10}$ and unusual high magnetic moments. ${ }^{5,8,15}$

In this study, we use UV-Vis $(2.0-5.5 \mathrm{eV})$ absorption spectroscopy as a tool to probe the electronic structure of small silver clusters. The challenge in such studies arises from the limited intensities of the cluster sources, further restricted by the size selection that rejects all clusters with the improper size. In the end only very low cluster densities are available for absorption experiments. To circumvent this problem, clusters can be accumulated in a transparent matrix, by codepositing the clusters and the matrix material over a period of time. Optical absorption measurements can then be performed on the resulting seeded matrix. Rare gases have long been used as matrix materials because of their transparency in the optical region resulting from their large band

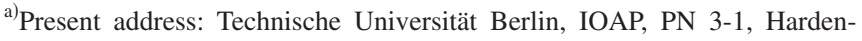
bergstraße 36, D-10623 Berlin, Germany.

b) Present address: Instituto de Física "Gleb Wataghin," UNICAMP, C.P. 6165, 13083-970 Campinas SP, Brazil.
}

gap $^{16,17}$ and due to their low interaction with the clusters. Moreover, in this situation the temperature of the clusters is well defined and low since the matrices condense at cryogenic temperature.

Optical absorption of small silver clusters $\left(\mathrm{Ag}_{1}\right.$ to $\left.\mathrm{Ag}_{39}\right)$ embedded in argon matrices has been studied previously ${ }^{3,4}$ but mainly for odd sized clusters. Due to the building mechanism and the high internal temperature in the sputter cluster source used, the cluster intensity decreases nearly exponentially. In addition even-sized clusters only have a low intensity because they are less stable due to electronic shellclosing effects. ${ }^{18}$ Measurements on other metal cluster systems have not been performed because of insufficient cluster intensities and lower cross sections.

We report here on a new absorption setup designed to record with increased sensitivity the absorption spectra of size-selected small metal clusters embedded in rare gas matrices. The design, construction, and performance of this setup are discussed and demonstrated in comparison with previously reported absorption spectra for $\mathrm{Ag}_{1}$ in argon. Also, we present the absorption spectra of $\mathrm{Ag}_{6}$ and $\mathrm{Ag}_{10}$, the least abundant silver clusters produced by our source for $\mathrm{Ag}_{n}, n \leqslant 39$.

\section{ABSORPTION EXPERIMENT}

Photoabsorption experiments on matrix isolated clusters are conceptually simple: light is injected through the rare gas matrix with embedded clusters and compared to a reference signal recorded on a matrix with no cluster inside. In the second case, the transmitted intensity $I_{0}$ characterizes the lamp intensity multiplied by the transmission of the whole optical system, the matrix scattering, and the detector efficiency, while in the first case, $I$ includes additionally the reduced transmission due to the absorption of the clusters. The absorption spectrum is analyzed using Beer's law, 


$$
I(\lambda)=I_{0}(\lambda) \exp \left[-\sigma(\lambda) \frac{N}{V} d\right],
$$

where $\sigma(\lambda)$ is the absorption cross section of a single cluster for a given wavelength $\lambda, N$ the total number of clusters in the matrix, $V$ the volume of the matrix, and $d$ the optical path length of the matrix.

It is important to notice that in optical absorption spectroscopy the signal is the reduction in the original light intensity due to its interaction with the analyzed system, therefore with dilute systems this results in a small signal on a high background. This is in contrast to other spectroscopic techniques (fluorescence, electron/ion yield), where the measurements consist in peaks added to an almost zero background. In consequence, the feasibility of the experiment is strongly dependent on having a high quality reference $I_{0}$ and a signal to noise ratio which is small compared to the absorption $I_{0}-I$.

From Eq. (1), the parameters responsible for the experimental sensitivity are the cluster density $N / V$ and the optical path length $d$. The density of size-selected clusters is limited by the production mechanism: even though it is imaginable to increase the amount of material deposited, in practice a deposition time exceeding a few hours is not feasible, because of the lack of reliability of a cluster source that cannot run unattended. In contrast a significant increase of sensitivity can be obtained by extending the optical path length $d$.

With this same cluster source, Fedrigo et al. ${ }^{3,19}$ used to accumulate clusters in argon matrices condensed on a $\mathrm{CaF}_{2}$ substrate for a few hours; the dimensions of the seeded matrix spot were $\sim 6 \mathrm{~mm}$ in diameter and $\sim 2 \mu \mathrm{m}$ thick. The absorption was then recorded by injecting the light through the $\sim 2 \mu \mathrm{m}$ matrix. To improve the sensitivity, the setup described in this article uses the following simple idea: increase the optical path by recording the absorption through the lateral dimension (millimeters) of the matrix rather than through its thickness (approximately in micrometers).

Similar setups have been used by other groups in the past, ${ }^{20-22}$ however, neon was used because its crystallization properties are easier to handle and optical paths of several centimeters then are possible. To understand the effect of the matrix material on the electronic structure of small metal clusters, we wanted to be able to perform absorption studies in different matrices such as argon. ${ }^{23}$ Argon tends to grow in nanocrystallites, leading to a severe limitation of the transparency of the matrices grown at low temperature. The optical path needs therefore to be shorter than in the experiments mentioned above. Simultaneously the thickness of the matrices has to be reduced at the expense of light transmission since the particle intensities available here are two orders of magnitude smaller. In the following, we describe the absorption device and its characterization.

\section{EXPERIMENTAL SETUP}

\section{A. Cluster source}

As the cluster source is described in detail elsewhere ${ }^{2}$ it will be only briefly discussed here. Clusters are generated by sputtering a metal target with a $15 \mathrm{~mA} \mathrm{Xe}{ }^{+}$ion beam at

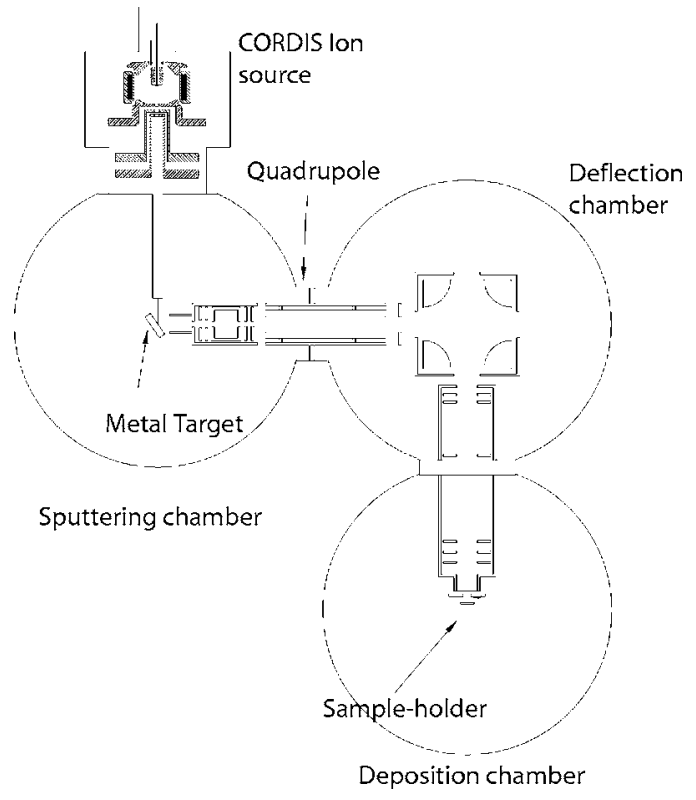

FIG. 1. Setup of the experiment. The clusters are produced in a cold reflex discharge ion source (CORDIS) sputtering source and are mass selected by a rf quadrupole. The cations are separated from the neutral clusters by a deflection quadrupole and guided to the sample holder through several sets of electrostatic lenses.

$25 \mathrm{keV}$ (see Fig. 1). The cluster ions resulting from the sputtering process are guided, using electrostatic lenses, to a quadrupole mass selector. The beam is then deflected by an electrostatic bending quadrupole to remove the neutral clusters from the beam. The bender also acts as an energy filter that removes the high energy ions resulting from the sputtering process. The remaining cations are guided to the sample holder. The temperature necessary for argon condensation is achieved by a closed-cycle cryostat.

To avoid beam spreading, the last lens element and the sample holder are kept at $5 \mathrm{~mm}$ from each other. This distance cannot be shorten since it is necessary to implement here a source of electrons for the cluster neutralization and the gas injector to grow the matrix (see Fig. 3).

Figure 2 shows a typical mass spectrum for $\mathrm{Ag}_{n}$ clusters recorded with our cluster source in the size range $n=1-15$. The intensity shows odd/even alternation that is due to electronic shell-closing effects. ${ }^{18}$ In particular, clusters with six and ten-atoms have the lowest intensities in the size range $n \leqslant 39$ (Ref. 4) and it is therefore difficult to perform experiments on these systems.

\section{B. Absorption device}

Figure 3 shows the absorption device designed to record the absorption along the length of the matrix, i.e., perpendicular to the deposition axis. The difficulty consists of making sure that the light travels through and only through the matrix. The setup is composed of a copper block connected to the cryostat, where matrix and cluster are deposited. The optical path is defined by two $40 \mu \mathrm{m}$ slits positioned at $2 \mathrm{~mm}$ distance. The slits are built by sections of $2 \times 2 \mathrm{~mm}^{2}$ of copper spaced from the block by a $40 \mu \mathrm{m}$ gold foil. This ensures the good thermal contact necessary to cool the whole device to ensure the condensation of the rare gas matrix in 


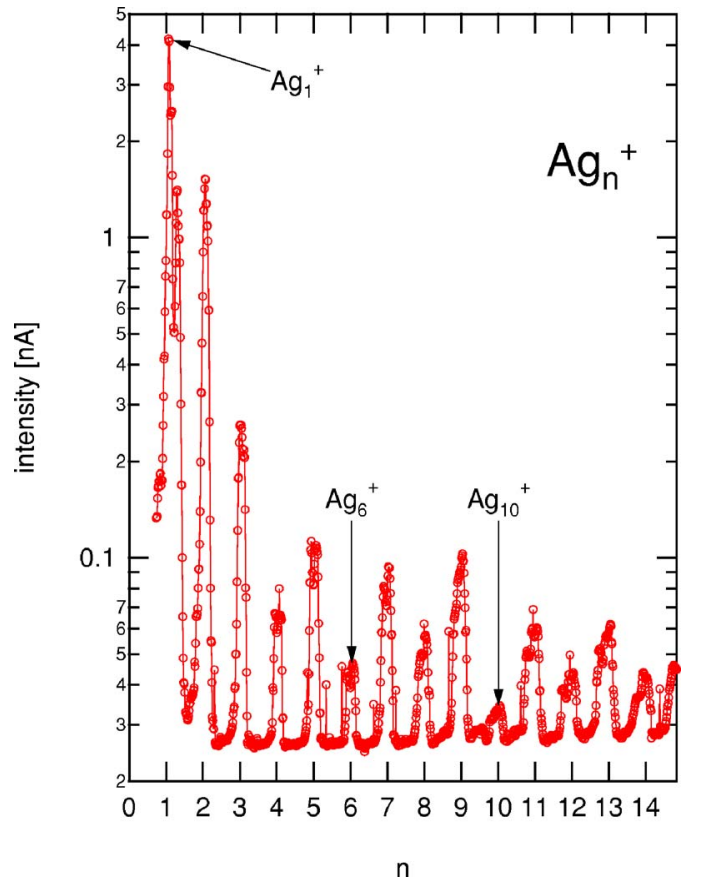

FIG. 2. (Color online) Mass spectrum of positively charged Ag clusters. The cluster current is measured on the sample and the deposition energy is $30 \mathrm{eV}$. Cluster sizes discussed in Sec. IV are signaled by arrows.

between the two slits. One of the slits is used to inject light in the matrix, while the other selects the light that has crossed the matrix.

The light produced by a deuterium or a tungsten lamp is focused by a spherical concave mirror and injected into the vacuum chamber through a sapphire view port. After crossing the matrix, the light is collected by an optical fiber and guided to the optical spectrometer $(0.75 \mathrm{~m}$ length). The light is dispersed by a $300 \mathrm{~nm}$ grating blazed at $250 \mathrm{~nm}$ and recorded by a liquid nitrogen cooled charge coupled device (CCD) camera, which allows for parallel data acquisition and long integration times.

\section{Sensitivity}

The longer the optical path, the higher the sensitivity; a long optical path, however, also implies strong losses due to light scattering on the argon nanocrystallites and therefore long acquisition times. The chosen path length of $2 \mathrm{~mm}$ is a result of a compromise between sensitivity and intensity.

An estimation of the sensitivity increase can be made considering Eq. (1) and comparing the geometry of our new setup to the previous one, which was already used successfully to measure UV-Vis absorption spectra of small metal clusters. The optical path is multiplied by 1000 from $2 \mu \mathrm{m}$ to $2 \mathrm{~mm}$. In the new version the matrix is thicker (40 $\mu \mathrm{m}$ compared to the previous $2 \mu \mathrm{m}$ ), therefore the volume $V$ of the matrix is increased by a factor of $\sim 20$. The total number of clusters $N$ does not change in both configurations. So, the gain in sensitivity can therefore be estimated to $1000 / 20=50$. This enhanced sensitivity by geometrical characteristic improvements allows us to measure systems with either a lower number of particles $N$ or a lower cluster absorption cross section $\sigma(\lambda)$. a)

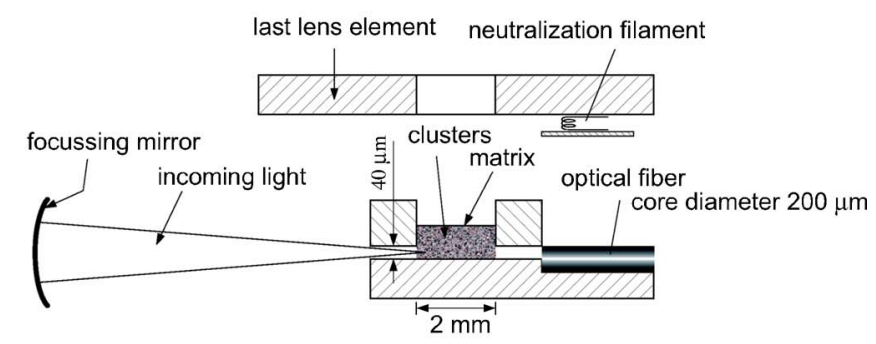

b)

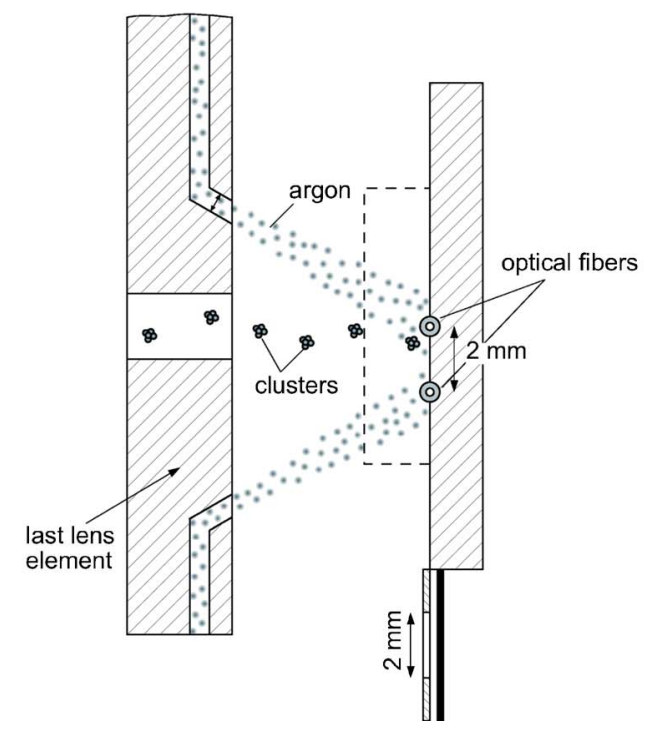

FIG. 3. Absorption device. (a) Horizontal cut. The argon matrix is condensed on a polished copper surface. Two small copper blocks are fixed on this surface to limit the optical path by two slits of $\sim 40 \mu \mathrm{m}$ width. (b) Vertical cut. Clusters are codeposited in the argon matrix and neutralized by electrons produced by a hot filament. Small pipes are bored inside the last lens element to inject the argon. A second optical fiber is placed off axis to record a reference spectrum.

\section{Intensity}

The total intensity recorded with this device is a function of the transmission of each component and the efficiency of the detector. The recorded intensity is therefore given by $I(\lambda)=I_{0}(\lambda) \cdot \Pi_{i} T_{i}(\lambda)$, where $I_{0}(\lambda)$ is the intensity of the light source at wavelength $\lambda$ and $T_{i}(\lambda)$ are the transmission/ efficiency coefficients of all the parts of the setup, namely, the optics used to inject the light in the sample holder, the view port, the absorption device itself, the optical fiber, the coupling optics to the spectrometer, the grating and mirrors of the spectrometer, and finally the efficiency of the CCD camera. The transmission of the argon matrix itself is discussed below. Figure 4 shows the transmission/efficiency of different parts of the setup as well as their product.

The transmission of the setup (Fig. 4) drops in the UV; this is further amplified by the scattering of the condensed argon matrix (see below). A diffraction grating blazed at $250 \mathrm{~nm}$ is therefore used in the spectrometer.

\section{E. Matrix transparency and uniformity}

As mentioned above, a critical point when using argon as matrix is its transparency to photons in the range of interest $(2-5.5 \mathrm{eV})$. Rayleigh scattering by the argon nanocrystallites strongly limits the sample transmission. 


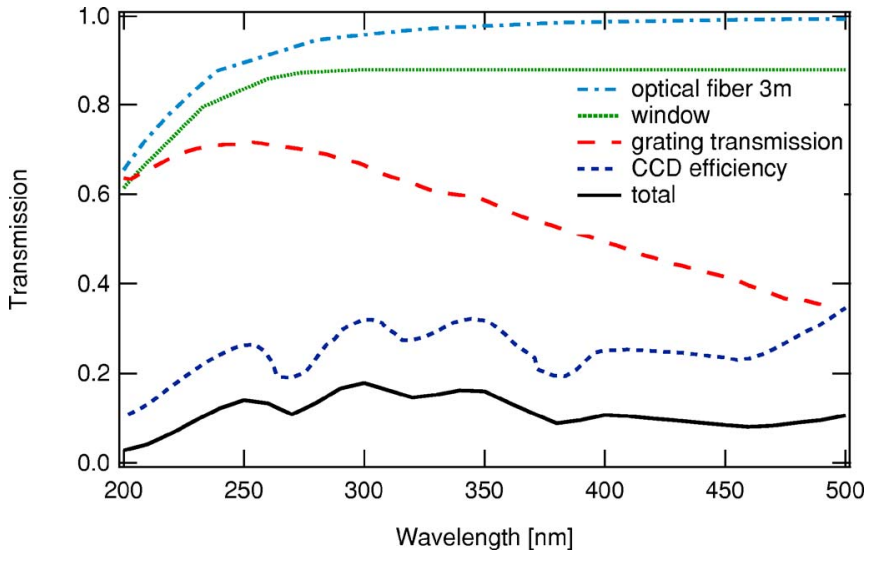

FIG. 4. (Color online) Transmission/efficiency of different parts of the setup as well as their product.

According to Fugol', ${ }^{24}$ when the condensation temperature of the argon matrix (here $T=28 \mathrm{~K}$ ) is higher than $2 / 3$ of its sublimation temperature $(\sim 30 \mathrm{~K})$, fcc grains of about $100 \mathrm{~nm}$ are obtained. These grains scatter the incoming light, resulting in poor transmission properties of the matrix. Since Rayleigh scattering scales with $1 / \lambda^{4}$ this problem is especially important in the UV region. We typically get a matrix transmission of $0.3 \%$ at $250 \mathrm{~nm}$.

The main parameters that control the matrix optical transmission are substrate cleanness, gas purity, growth velocity, and temperature. The cleanness of the substrate surface was found to influence strongly the matrix transmission. To ensure a clean substrate, the following procedure was implemented: starting from a temperature of $\sim 30 \mathrm{~K}$, the sample holder was flashed to $280 \mathrm{~K}$, to evaporate any possible condensed gas, it was then cooled as fast as possible, and the deposition started as soon as the substrate reached the condensing temperature of argon.

The growth rate is set by the time of the deposition and the minimal matrix thickness $(40 \mu \mathrm{m})$ defined by the slits.

The homogeneity of the matrix is further improved by controlling the injection of the rare gas. It was found that this is a crucial point in the design and careful attention was given to the conception of the two orifices in the last lens element that inject the rare gas towards the substrate. Each orifice has a diameter 2.5 times larger than the diameter of the optical fiber to insure a homogenous growth of the part of the matrix seen by the fiber and a separate injector points to the center of each optical fiber [see Fig. 3(b)].

Optical interferences produced by the laser beam reflected from the matrix surface and the copper surface are used to monitor the matrix growth. The time interval between two maxima in the oscillating interference signal gives the growth velocity $(\lambda / 2) / \Delta t$, where $\lambda=632.8 \mathrm{~nm}$ is the wavelength of the HeNe laser used. The number of visible oscillations is a signature of the optical quality. A typical example is shown in Fig. 5, where the interferences during a typical deposition are shown. The argon flux was calibrated to grow the argon matrix at a rate of $16 \mu \mathrm{m} / \mathrm{h}$. At the end of the cluster deposition a capping layer of $30 \mu \mathrm{m}$ was added to protect the clusters and to enhance the light coupling into the matrix.

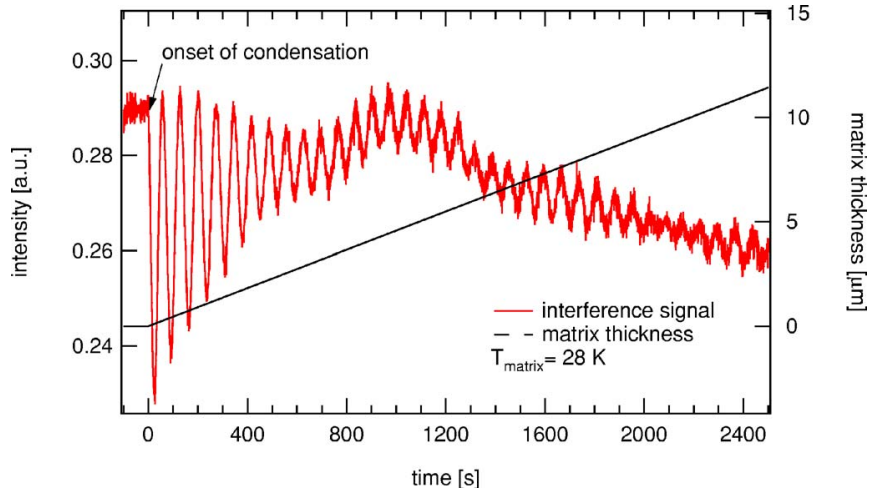

FIG. 5. (Color online) Interferometric monitoring of the growth of the matrix. The oscillations in the reflected laser beam (left scale) are seen throughout the $2 \mathrm{~h}$ long deposition, while the matrix thickness is increasing linearly (right scale).

\section{RESULTS}

We report here the absorption measurements of different samples performed to characterize the new absorption device. $\mathrm{Ag}_{1}$ is reported for comparison with previously reported spectra and for an experimental determination of sensitivity gain obtained with the setup. The absorption spectra of $\mathrm{Ag}_{6}$ and $\mathrm{Ag}_{10}$ are then given as demonstration of the power of the method since these are the two cluster sizes with the lowest intensities in the mass spectrum.

\section{A. Sensitivity increase: $\mathbf{A g}_{1}$ in argon}

For the previously used monomer density of $95 \mathrm{nA} \mathrm{h} \mathrm{cm}^{-2}$, the sensitivity increase with the new absorption device resulted in a complete saturation of the absorption signal for the monomer, the deposition current had to be limited to avoid this. The absorption spectrum shown in Fig. 6(a) results from the logarithm of the ratio of the reference $I_{0}$ and signal $I$ [Eq. (1)]. The reference $I_{0}$ can either be recorded through the second optical fiber or in a separate experiment with the same fiber but in a matrix with no cluster. Figure 6(b) reports the absorption spectrum recorded by Fedrigo ${ }^{19}$

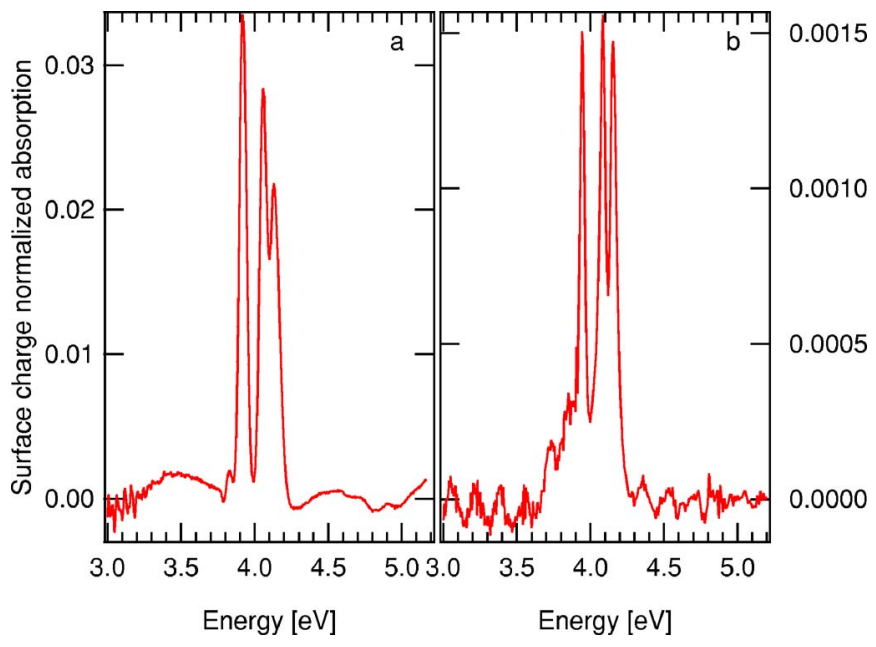

FIG. 6. (Color online) Absorption spectra of $\mathrm{Ag}_{1}$ recorded with (a) the new absorption setup compared to (b) the absorption of the same system recorded by Fedrigo (Ref. 19). The signal has been normalized by the surface charge in $\left(\mathrm{nA} \mathrm{h} \mathrm{cm}^{-2}\right)$ for comparison purposes. Sensitivity increase: $\times 20$. 
for comparison; the intensity scale has been corrected by the monomer surface density estimated from the deposition parameters. The deposition by Fedrigo ${ }^{19}$ has been normalized to $95 \mathrm{nA} \mathrm{h} \mathrm{cm}{ }^{-2}$, while in our measurement the monomer ion surface density was $18 \mathrm{nA} \mathrm{h} \mathrm{cm}^{-2}$.

One first notices the excellent agreement between the two spectral signatures; both the peak positions and their widths are identical.

A sensitivity increase of a factor of $\sim 20$ is demonstrated with the new absorption device. This is in mild agreement with the estimated factor of 50. The discrepancy between these two values has, however, to be put in perspective with the assumptions made for the intensity calibration. While the current density is measured precisely in our case [cluster current measured through a $2 \mathrm{~mm}$ hole, as shown at bottom of Fig. 3(b)], it is more difficult to estimate for the deposition by Fedrigo et al. since it is based on a total current measurement and a visual estimation of the particle spot diameter of $6 \mathrm{~mm}$. A small overestimation of this value would have a considerable effect on the intensity calibration. With this in mind, we can consider the agreement of the measured value with the estimated value as reasonable.

The oscillations due to interferences from reflections from the surfaces of the matrix in the $2 \mu \mathrm{m}$ thick matrix [Fig. 6(b)] are absent in Fig. 6(a) because the optical path is now much longer than the wavelength of the light. This also adds to the quality of the absorption spectrum [Fig. 6(a)].

\section{B. Absorption spectra of least abundant clusters}

To demonstrate the performance of the absorption device we have recorded the absorption spectra of the two least abundant clusters in our cluster source in the small size range $n \leqslant 39$, namely, $\mathrm{Ag}_{6}$ and $\mathrm{Ag}_{10}$ (Fig. 2). Clusters have been accumulated in the matrix for $2.5 \mathrm{~h}$ with a current of $25 \mathrm{pA}$ for $\mathrm{Ag}_{6}$, respectively, $18 \mathrm{pA}$ for $\mathrm{Ag}_{10}$, corresponding to cluster ions fluxes of $0.8 \mathrm{nA} \mathrm{cm}^{-2}$, respectively, $0.6 \mathrm{nA} \mathrm{cm}^{-2}$, and cluster densities in the matrix of $1 \times 10^{16}$ and 8 $\times 10^{15} \mathrm{~cm}^{-3} . \mathrm{Ag}_{6}$ shows several clear transitions: a sharp intense double peak at 3.59 and $3.65 \mathrm{eV}$, a second double peak at 4.10 and $4.17 \mathrm{eV}$ with a left shoulder, and finally two peaks at 4.91 and $5.13 \mathrm{eV}$. For $\mathrm{Ag}_{10}$, we observe three main peaks at $3.78,3.97$, and $4.15 \mathrm{eV}$. The lower intensity peaks below $3.5 \mathrm{eV}$ are most probably experimental artifacts as it is apparent when comparing the spectra for $\mathrm{Ag}_{6}$ and $\mathrm{Ag}_{10}$ (Fig. 7).

These experiments show for the first time the absorption spectra for these cluster species, with very distinct features, and therefore demonstrate clearly the capabilities of our new setup.

As mentioned above, the low intensities of these two species are due their lower stability compared to the other cluster sizes. ${ }^{18}$ Therefore their surviving rate upon deposition may be smaller than for the other cluster sizes as well. The absorption spectrum of $\mathrm{Ag}_{6}$ does not show similarities with the absorption of $\mathrm{Ag}_{5}$, indicating that the fragmentation is small. For $\mathrm{Ag}_{10}$, we probably see some fragmentation since the spectrum has some similarities with the one of $\mathrm{Ag}_{9}$. The

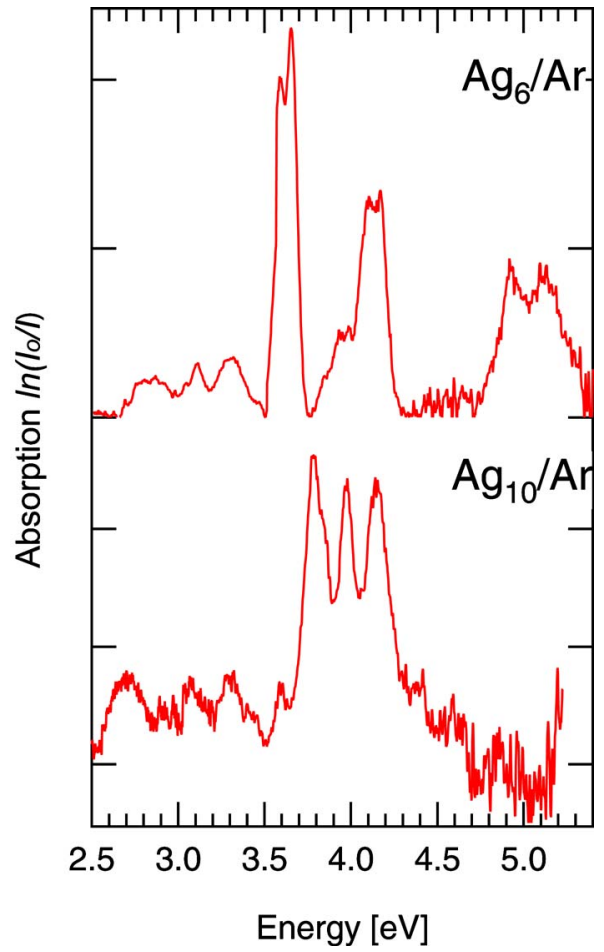

FIG. 7. (Color online) UV-Vis absorption spectra of $\mathrm{Ag}_{6}$ and $\mathrm{Ag}_{10}$ in argon.

details of the measurements and their interpretation will be discussed elsewhere.

\section{DISCUSSION}

A new device for optical absorptions of small clusters deposited in an argon matrix is described and its efficiency is evaluated on silver atoms in argon. An enhanced efficiency of $\sim 20$ is demonstrated. To further show the performance of the system, absorption spectra of the two least abundant silver cluster ion sizes from the cluster source in the small size regime that had not been measured up to now are reported. The ability to study highly diluted samples with densities below $8 \times 10^{15} \mathrm{~cm}^{-3}$ is also demonstrated. The performance increase offers the possibility of optical UV-Vis absorption investigations of systems (such as $\mathrm{Au}, \mathrm{Cu}, \mathrm{Ni}$, etc.) not studied up to now because of both lower intensities in the cluster ion yield and lower absorption cross sections.

${ }^{1}$ W. A. de Heer, K. Selby, V. Kresin, J. Masui, M. Vollmer, A. Chatelain, and W. D. Knight, Phys. Rev. Lett. 59, 1805 (1987).

${ }^{2}$ W. Harbich, S. Fedrigo, F. Meyer, D. M. Lindsay, J. Lignieres, J. C. Rivoal, and D. Kreisle, J. Chem. Phys. 93, 8535 (1990).

${ }^{3}$ S. Fedrigo, W. Harbich, and J. Buttet, Phys. Rev. B 47, 10706 (1993).

${ }^{4}$ W. Harbich, S. Fedrigo, and J. Buttet, Z. Phys. D: At., Mol. Clusters 26, 138 (1993).

${ }^{5}$ I. M. L. Billas, A. Chatelain, and W. A. de Heer, Science 265, 1682 (1994).

${ }^{6}$ A. Sanchez, S. Abbet, U. Heiz, W.-D. Schneider, H. Häkkinen, R. N. Barnett, and U. Landman, J. Phys. Chem. 103, 9573 (1999).

${ }^{7}$ C. Félix, C. Sieber, W. Harbich, J. Buttet, I. Rabin, W. Schulze, and G. Ertl, Phys. Rev. Lett. 86, 2992 (2001).

${ }^{8}$ J. T. Lau, A. Fohlisch, R. Nietubyc, M. Reif, and W. Wurth, Phys. Rev. Lett. 89, 057201 (2002).

${ }^{9}$ C. Sieber, W. Harbich, J. Buttet, C. Félix, R. Mitrić, and V. BonačićKoutecký, Phys. Rev. A 70, 041201 (2004).

${ }^{10}$ B. Yoon, H. Hakkinen, U. Landman, A. S. Worz, J.-M. Antonietti, S. Abbet, K. Judai, and U. Heiz, Science 307, 403 (2005). 
${ }^{11}$ Metal Clusters, Wiley Series in Theoretical Chemistry, edited by W. Ekardt (Wiley, Chichester, 1999).

${ }^{12}$ B. von Issendorff and O. Cheshnovsky, Annu. Rev. Phys. Chem. 56, 549-580 (2005).

${ }^{13}$ L. A. Peyser, A. E. Vinson, A. P. Bartko, and R. M. Dickson, Science 291, 103 (2001).

${ }^{14}$ T. H. Lee, J. I. Gonzales, and R. M. Dickson, Proc. Natl. Acad. Sci. U.S.A. 99, 10272 (2002).

${ }^{15}$ P. Gambardella et al., Science 300, 1130 (2003).

${ }^{16}$ M. Klein and J. Venables, Rare Gas Solids (Academic, New York, 1977),

Vol. II.

${ }^{17}$ M. Jacox, J. Phys. Chem. Ref. Data 27, 115 (1998).
${ }^{18}$ W. A. de Heer, Rev. Mod. Phys. 65, 611 (1993).

${ }^{19}$ S. Fedrigo, Ph.D. thesis, EPFL, 1992 (http://library.epfl.ch/theses/?nr $=1084$ ).

${ }^{20}$ R. Rossetti and L. E. Brus, Rev. Sci. Instrum. 51, 467 (1980).

${ }^{21}$ V. E. Bondybey, T. A. Miller, and J. H. English, J. Chem. Phys. 72, 2193 (1980).

${ }^{22}$ S. Leutwyler, J. P. Maier, and U. Spittel, Chem. Phys. Lett. 96, 645 (1983).

${ }^{23}$ F. Conus, V. Rodrigues, S. Lecoultre, A. Rydlo, and C. Félix, J. Chem. Phys. 125, 024511 (2006).

${ }^{24}$ I. Y. Fugol', Adv. Phys. 27, 1 (1978). 